\title{
Aerial inventory of fragmented forest using airborne hyperspectral imaging
}

\begin{abstract}
Due to heavy development at Kuala Lumpur, Bukit Sungai Puteh Forest Reserve had been fragmented and degraded requiring a precision approach management which demands a comprehensive assessment of the area. This study was to evaluate the forest resource with higher accuracy through airborne hyperspectral and subsequently mapping the tree species distribution. In this study, hyperspectral sensor was flown at an altitude of 1,000m to acquire imagery with spatial resolution of $1.0 \mathrm{~m} \times 1.0 \mathrm{~m}$. Imagery was processed and subsequently classified using Spectral Angular Mapper classification. Data then transformed into Geographic Information System mode to generate crown mapping and tree mapping. Results obtain revealed that there were 1,484 trees of above $25 \mathrm{~cm}$ diameter comprising 24 species in this area. Based on 90 randomly selected trees, the accuracy was found to be $72 \%$. From this study, it can be concluded that hyperspectral remote sensing is acceptably accurate in assessing fragmented forest.
\end{abstract}

Keyword: Aerial inventory; Mapping; Disturbed fragmented forest; Airborne hyperpsectral 